\title{
Effects of Exercise on Physical Fitness and Strength According to the Frailty Level of Female Elderly with Hypertension
}

\author{
Kyung-Wan Baek ${ }^{*}$ PhD, Min Chul Lee ${ }^{2 *}$ PhD, Tae-Bong Jeon ${ }^{1}$ MS, Jun-Il Yoo ${ }^{3}$ MD, PhD, Jin Sung Park ${ }^{3}$ MD, PhD, \\ Hyo Youl Moon ${ }^{4} \mathrm{PhD}$, Ji-Seok $\mathrm{Kim}^{1} \mathrm{PhD}$ \\ 'Department of Physical Education, Gyeongsang National University, Jinju; ${ }^{2}$ Department of Sports Medicine, CHA University, Pocheon; ${ }^{3}$ Department of Orthopaedic \\ Surgery, Gyeongsang National University Hospital, Jinju; ${ }^{4}$ Department of Physical Education, Seoul National University, Seoul, Korea
}

PURPOSE: The purpose of this study was to determine the effects of exercise on physical fitness and strength according to their frailty level of the hypertensive female elderly aged over 65.

METHODS: Female elderly subjects ( $>65$ year) with hypertension $(\mathrm{n}=90)$ were classified according to their level of frailty (Fr_), and were divided into control group (Ctrl) and exercise group (Ex). Hypertensive female elderly in exercise groups were subjected to 8 -week combined exercise of aerobic (stretching and walking) and resistance (elastic band and weight training) exercise twice per week (50 minutes $\times 2$ times $\times 8$ weeks). Body composition, functional fitness and skeletal muscle strength were evaluated as main variables (Ctrl, n=27; Ex, n=23; Fr_Ctrl, n=17; Fr_Ex, n=23).

RESULTS: Body composition, blood pressure, fasting glucose level (include hemoglobin $\mathrm{A}_{1 \mathrm{c}}$ ) and blood lipid profile did not interact with the exercise intervention and frailty level. Records of 30-second chair stretch test, two-minute walk test and arm curl increased after exercise intervention regardless of frailty level. The strength of quadriceps femoris increased after exercise intervention regardless of the level of frailty. However, the strength of biceps femoris $(p<.001)$, biceps brachii $(p<.05)$ and triceps brachii $(p<.001)$ was significantly increased in the Fr_Ex compared to pre-intervention of exercise.

CONCLUSIONS: The classification according to the level of frailty in hypertensive female elderly can have a significant effect on the degree of muscle strength improvement by exercise intervention. In addition, this study newly discovered that the improvement of 30-s chair stretch test record in the hypertensive elderly women appeared faster than the improvement of the rapid-gait test record independently of the level of frailty.

Key words: Frailty, Hypertension, Female, Elderly, Combined exercise, Fitness

\section{INTRODUCTION}

Despite the fact that the incidence of hypertension at a certain age has little changed, the number of elderly hypertensive patients is increasing exponentially due to the aging of the population [1]. According to observational data form Framingham study, the lifetime risk of developing hypertension between 55 and 65 years of age is greater than 90\% [2]. Hence, the ever-increasing number of elderly people ultimately leads to an increase in hypertensive population. Since most of the elderly are at high risk of hypertension, it is thought that the exclusion of healthy elderly subject without hypertension from the study subjects in clinical studies reflects the characteristics of the elderly population group well. In particular, the inclusion of non-hypertensive elderly subjects in the study in exercise-related studies can be cause to underestimation the degree of improvement of specific physical factors after exercise-intervention. The prevalence of hypertension in Korea is higher in men than in women before age 60 , but after age 60 , women have a higher prevalence than men, and the difference increases with age [3]. Therefore, the popu-

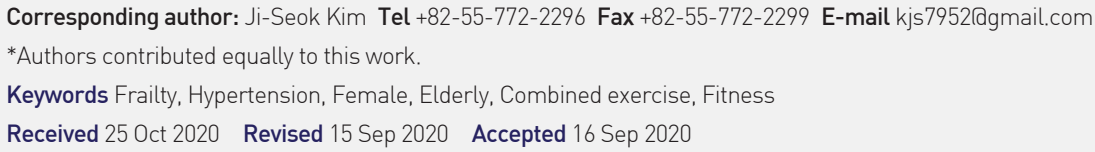


lation group most susceptible to hypertension in Korea is the elderly women. In summary, excluding healthy subjects without hypertension and male subjects among elderly 65 years or older may be the minimum criterion for screening the most vulnerable population in the elderly.

As mentioned above, the most vulnerable population in the elderly is female hypertensive patients. Therefore, exercise intervention studies targeting female hypertension patients are still being actively conducted [46]. Nevertheless, there has been no study in which elderly women with hypertension were classified according to frailty criteria and exercise intervention was conducted. If the difference in exercise intervention in elderly women with hypertension varies according to the level of frailty, additional considerations in exercise intervention studies can be suggested. It is assumed that the elderly women with hypertension as the subject of the study are the weakest population in terms of physical fitness. However, elderly women with hypertension may also have different level of frailty. If there is a difference when exercise intervention is performed in consideration of the additional classification of hypertensive females according female elderly according to their level of frailty, it can be a good basis for suggesting important considerations when exercise prescriptions are required for the elderly with hypertension. In addition, the analysis of blood pressure (BP), blood lipid profiles, fasting glucose level and hemoglobin $\mathrm{A}_{\mathrm{lc}}\left(\mathrm{Hb}_{\mathrm{lc}}\right)$ were also conducted to examine whether the change in physical fitness and strength according to the level of frailty in the elderly women with hypertension could be caused by the preceding change of other physiological factors. BP, blood lipid profiles, fasting glucose level and $\mathrm{HbA}_{\mathrm{lc}}$ analysis were also aimed at providing feedback to all participants on whether or not positive changes in hypertension, insulin resistance, and blood lipid profiles were caused by exercise intervention.

We hypothesized that even in elderly women with hypertension, differences in physical fitness factors after exercise intervention would be evident according to the level of frailty. The purpose of this study was to classify the elderly women with hypertension according to their level of frailty, then performed exercise intervention and check whether physical fitness differ according to the level of frailty.

\section{METHODS}

\section{Participants}

Participants responded to advertisement through a local public health center and were screened by phone or consultation in person. All candidates who visited the local public healthcare center had their BP measured, and a frailty-related physical fitness test (rapid-gait test, RGT; 30-s sitting and standing up in a hardback chair, 30-s CST) was performed [7]. Most of the women who participated in the study had BP of 140 $\mathrm{mmHg}$ or more in systolic or $90 \mathrm{mmHg}$ in diastolic BP [8], and very few non-hypertensive subjects were excluded from the study. If the RGT record was more than 10 seconds or the number of repetitions of 30-s CST was 0 , the patient was identified as a frailty person. According to the aforementioned criterion for determining frailty, it was classified into normal and frailty (Fr_), and subjects to perform to the exercise (Ex) were assigned randomly (Ctrl, n=27; Ex, n=23, Fr_Ctrl, n=17, Fr_Ex, $\mathrm{n}=23$ ). Body composition, functional fitness test, strength, BP, fasting glucose level, and $\mathrm{HbAlc}$ were measured before and after exercise intervention, respectively, and data were collected. The collected data was statistically analyzed. The characteristics of the study subjects are shown in Table 1.

All participants were over 65 years old and were non-smokers with no history of coronary artery disease (Fig. 1). All participants provided written consent after being provided with sufficient information about the study objectives, procedures and risks. They were asked to maintain their current lifestyle for an eight-week intervention period. The protocol was approved by the Institutional Review Board of Gyeongsang National University Hospital (IRB No. GNUH 2015-02-001).

\section{Study methods and exercise training protocols}

8-weeks combined exercise was carried out twice per week, with each session consisting of 50 minutes according to our laboratory exercise

Table 1. Subject characteristics

\begin{tabular}{|c|c|c|c|c|c|}
\hline & Ctrl $(n=27)$ & $\operatorname{Ex}(n=23)$ & Fr_Ctrl $(n=17)$ & Fr_Ex $(n=23)$ & $p$ value \\
\hline Age (yr) & $71.72 \pm 6.31$ & $74.65 \pm 5.70$ & $78.47 \pm 6.25$ & $77.75 \pm 5.77$ & N.S. \\
\hline Height (cm) & $145 \pm 5.89$ & $145.47 \pm 5.60$ & $150.77 \pm 5.35$ & $147.67 \pm 5.52$ & N.S. \\
\hline Body weight (kg) & $56.50 \pm 7.85$ & $55.80 \pm 5.17$ & $51.43 \pm 12.46$ & $54.42 \pm 7.25$ & N.S. \\
\hline Body mass index $\left(\mathrm{kg} / \mathrm{m}^{2}\right)$ & $25.77 \pm 2.65$ & $23.94 \pm 2.10$ & $22.54 \pm 4.94$ & $24.64 \pm 3.36$ & N.S. \\
\hline Systolic blood pressure (mmHg) & $136.00 \pm 17.01$ & $132.05 \pm 12.46$ & $139.63 \pm 18.41$ & $129.58 \pm 12.29$ & N.S. \\
\hline Diastolic blood pressure (mmHg) & $79.62 \pm 10.17$ & $78.19 \pm 9.06$ & $80.06 \pm 9.90$ & $76.67 \pm 9.12$ & N.S. \\
\hline
\end{tabular}


protocol. The perception of exercise was monitored verbally on every session with the use of the Borg's rating of perceived exertion scale. Participants were allowed to pause or skip a set of exercises if they felt a sense of intense fatigue. Participants in the Ctrl and Fr_Ctrl were asked to maintain their current lifestyle during the experimental period. Before and after the exercise intervention, body compositions were measured by bioelectrical impedance analysis (Inbody 420, InBody Co., Ltd, Cheonan, Korea). Combined exercise consisted of 5-10 minutes of preparation exercise including stretching, 30-40 minutes of aerobic exercise

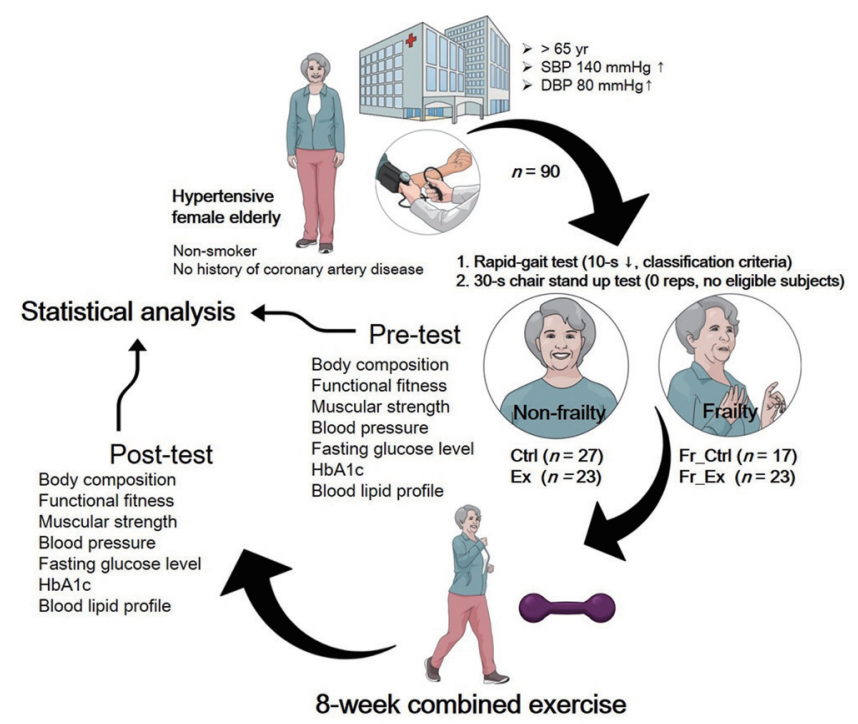

Fig. 1. Summary of study flow. such as walking on the ground, 30-40 minutes of resistance exercise using a slave band and 5-10 minutes of exercise training. In the case of resistive exercise, the upper body mainly used elastic band, while the lower body focused on the body movement.The detailed contents of the combined exercise program are shown in Table 2. To ensure safety, certified physicians supervised all exercise sessions.

\section{Functional fitness test}

We measured the functional fitness by using a very slightly self-modified senior fitness test [9]. Measurement and evaluation items methods were organized as follows. 1) The rapid-gait test (also known as 8-foot up and go). For measuring agility and dynamic balance. Start by sitting on a hard back chair and return as soon as possible along the $3 \mathrm{~m}$ course walk to sit in the chair where the subject started. 2) 30-s CST. Lower body strength was measured the number of repetitions of chair sit and stands for 30-s. 3) Arm curl. The upper body strength were measured on both arms. When the elbows is fully extended, bending up to $90^{\circ}$. While sitting chair, repeat with $2 \mathrm{~kg}$ dumbbell for 30-s with maximum effort. 4) The two-minute walk test. Which measures endurance, measures the number of repetitions the knee reaches the midpoint between the patella and the iliac crest when a 2-minute walk is performed. 5) Chair stretch test (chair sit and reach). Sitting on a chair measuring the flexibility of the lower leg, sits at the edge of the chair and stretches subjects leg without bending knees, allowing hands to slowly cling to toes. At this time

Table 2. A combined exercise program conducted by female elderly with hypertension in this study

\begin{tabular}{|c|c|c|c|c|}
\hline Components & Detailed program & Reps or time of detailed program & No. of set repetitions & Time of program \\
\hline Warm-up & Stretching & All joint Each $10 \mathrm{sec}$ & 1 rep & $5 \min$ \\
\hline Aerobic exercise & Walk in place & $1-2 \mathrm{~min}$ & 5-10 reps & $10 \min$ \\
\hline Resistance exercise (elastic band) & $\begin{array}{l}\text { Shoulder press } \\
\text { Front raise } \\
\text { Bent-over lateral raise } \\
\text { Kickback } \\
\text { Arm curl } \\
\text { Overhead triceps extension } \\
\text { Bent-over row } \\
\text { Seated row }\end{array}$ & $\begin{array}{l}\text { 1-4 week: } 15-20 \text { reps } \\
\text { 5-8 week: } 15-30 \text { reps }\end{array}$ & $3-5$ reps & $20-30 \mathrm{~min}$ \\
\hline Resistance exercise (calisthenics) & $\begin{array}{l}\text { Squat } \\
\text { Wide-stance squat } \\
\text { Half lunge } \\
\text { Donkey kicks } \\
\text { Hip extension } \\
\text { Standing calf raises } \\
\text { Toe raises } \\
\text { Wall push ups }\end{array}$ & $\begin{array}{l}\text { 1-4 week: } 10-15 \text { reps } \\
\text { 5-8 week: } 15-20 \text { reps }\end{array}$ & $3-5$ reps & $20-30 \mathrm{~min}$ \\
\hline Cool-down & Stretching & All joint Each $20 \mathrm{sec}$ & $1-3$ reps & $5-10 \mathrm{~min}$ \\
\hline
\end{tabular}


the distance between fingertips and toes (both right and left legs). 6) Back scratch. Back scratching was performed to measure the flexibility of the upper limb. The subject did their best to bring the fingertips of both hands closest on the back. At this time, the distance between both fingers was measured. To ensure safety, certified physicians supervised all exercise sessions.

\section{Measurement of muscular strength}

The strength of skeletal muscles were measured by dynamometer (Micro FET2, Hoggan Scientific, Salt Lake City, UT, USA). The reliability and reproducibility of this device have been tested in previous studies $[10,11]$. Muscular strength measurements were expressed as kilogramforce $(\mathrm{kg} \cdot \mathrm{f})$. We measured the quadriceps femoris, biceps femoris, biceps brachii and triceps brachii. All participants were instructed to sit with their legs suspended over the end of an examination table. Hips and knees were flexed to $90^{\circ}$ before the measurement. The height of the examination table was modified based on the participant's leg length to produce a distance of $10 \mathrm{~cm}$ between the feet and the floor. They were instructed to hold the side-edges of the examination table with their hands. After several trials with $<50 \%$ force as a warm-up, they were asked to carry out a maximal isometric voluntary contraction for $5 \mathrm{~s}$. For each test, participants carried out three isometric maximal voluntary contractions. A 1-min recovery period was provided to participants between each bout of contraction. Grip strength was measured with a digital grip strength dynamometer (TKK-5401, Takei Scientific Instruments Co., Ltd, Niigata, Japan). During the assessment, participants were asked to stand upright with their feet hip-width apart and to look forward with the elbow fully extended. The dynamometer was held by the testing hand in a neutral, comfortable position (not flexed or extended) with $90^{\circ}$ flexion at the index finger. Participants performed two trials for dominant hand. Participants were instructed to squeeze the grip continuously with full force for at least 3 seconds. The time between each trail was approximately 60 seconds. Among the measure results, a higher hand grip force $(\mathrm{kg} \cdot \mathrm{f})$ was recorded [12].

\section{Measurement of blood pressure, fasting glucose level and blood lipid profile}

$\mathrm{BP}$, fasting glucose level, and $\mathrm{HbA}_{\mathrm{lc}}$ were measured and analyzed by registered physician and health care provider in accordance with the public health center's own regulations. Blood was taken using blood sampling rod from the fingertip to analyze total cholesterol, low- and high-density lipoprotein cholesterol and triglycerides. The collected blood was dropped on an analysis cassette and then inserted into a blood analyzer (cholesterol LDX ${ }^{\circledR}$, Hayward, CA, USA) to analyze the blood lipid profiles.

\section{Statistical analysis}

It was confirmed that there was no difference between the groups before exercise intervention by performing a one-way ANOVA. Post-hoc test was performed by Tukey's multiple comparison test. Two-way ANOVA was performed for all measurement variables to determine whether there was an interaction between the intervention period and the group, and additionally, a multiple t-test was performed to confirm statistically significant differences. Post-hoc test was performed by Sidak's multiple comparisons test. GraphPad Prism (Version 8.3.0, GraphPad Software, La Jolla, California, USA) was used for all statistical analysis and plotting graph. The results were considered significant at $p<.05$.

\section{RESULTS}

\section{Body composition}

There were no significant differences in body mass index, waist to hip ratio, body fat mass, percentage body fat and skeletal muscle mass (Fig. 2).

\section{Fitness level}

The number of repetitions of 30-s CST significantly increased after exercise intervention in both Ex (pre vs. post, $p<.01$ ) and Fr_Ex (pre- vs. post, $p<.0001$ ) (Fig. 3B). Two-minute walk test records significantly increased after exercise intervention in both Ex (pre vs. post, $p<.0001$ ) and Fr_Ex (pre vs. post, $p<.0001$ ) compared to before (Fig. 3D). The number of repetitions of arm curl increased after exercise intervention in both Ex (pre vs. post, $p<.0001$ ) and Fr_Ex (pre vs. post, $p<.0001$ ) compared to before exercise intervention (Fig. 3E). In the RGT, chair stretch test, back scratch test there was no interaction between frailty and intervention, and there was no significant difference pre- and post-intervention (Fig. $3 \mathrm{~A}, \mathrm{C}$, and F).

\section{Muscular strength}

The quadriceps femoris strength increased significantly after exercise intervention in both Ex (pre vs. post, $p<.01$ ) and Fr_Ex (pre vs. post, $p<.001$ ) compared to before exercise intervention (Fig. 4A). However, the strength of biceps femoris, biceps brachii and triceps brachii increased 

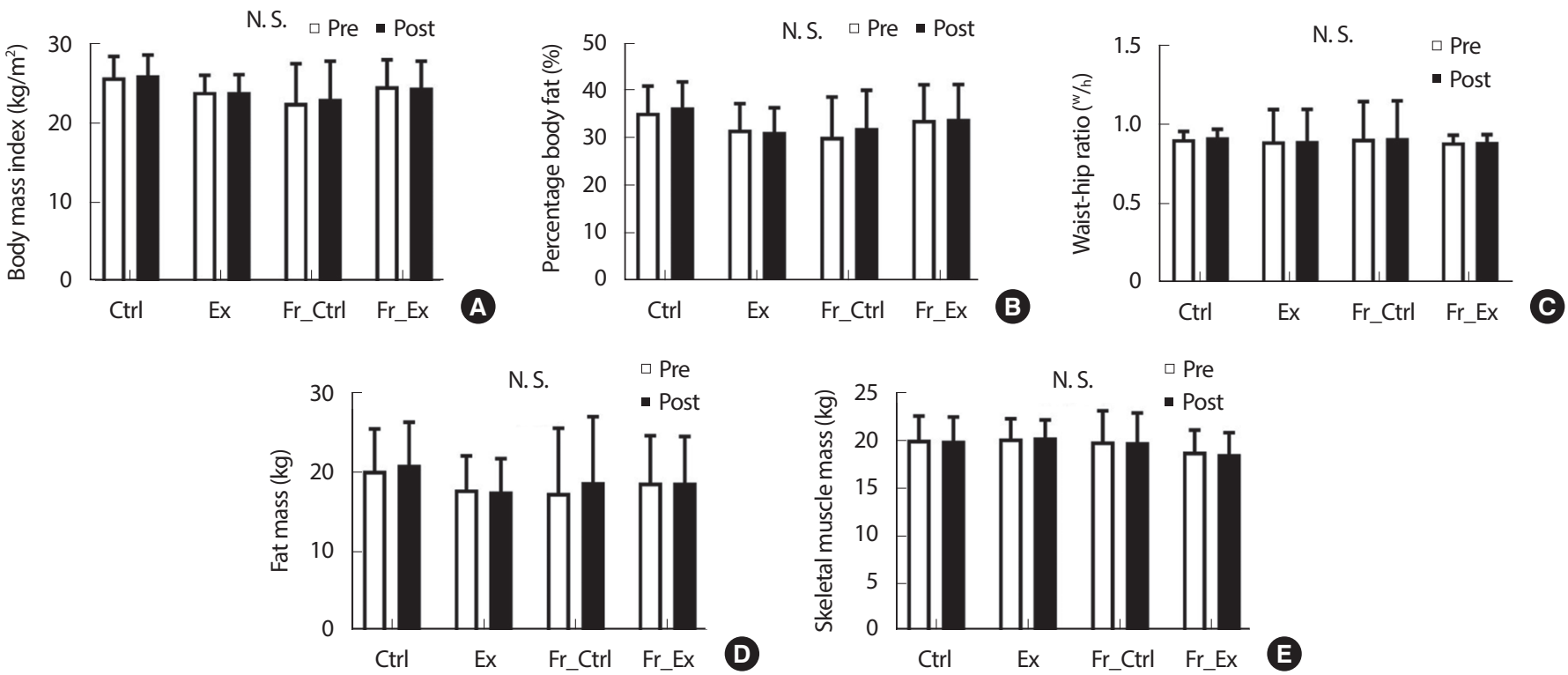

Fig. 2. Body composition of pre- and post-exercise intervention in hypertensive female elderly. Data are presented mean \pm standard deviation (SD). N. $\mathrm{S}$. $=$ no significant difference. $\mathrm{W} / \mathrm{H}=$ waist circumference/hip circumference.
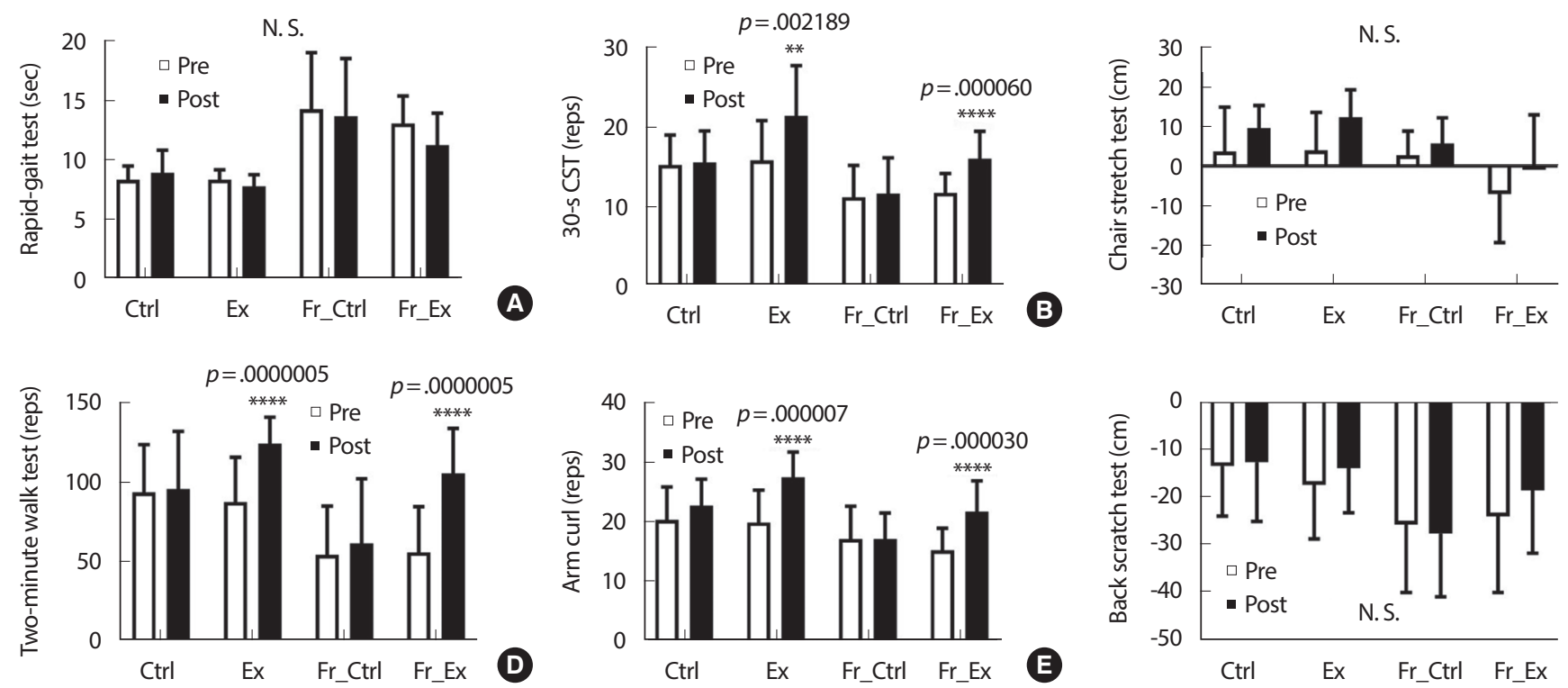

Fig. 3. Functional fitness of pre- and post-exercise intervention in hypertensive female elderly. Data are presented mean \pm SD. N. S. $=$ no significant difference. ${ }^{* *} p<.01$ and ${ }^{* *} p<.0001$, pre vs. post.

after exercise intervention compared to before exercise intervention only in Fr_Ex (biceps femoris, $p<.001$; biceps brachii, $p<.05$; triceps brachii, $p<.0001$ ) (Fig. 4B, C, and D). Grip strength had no interaction between exercise intervention and frailty level (Fig. 4E).

\section{Blood pressure, blood glucose and blood lipid profile}

There was no significant difference in BP, fasting blood glucose level (including $\mathrm{HbAlc}$ ) and blood lipid profile before and after intervention
(Fig. 5).

\section{DISCUSSION}

The purpose of this study was to determine whether the classification by frailty level of elderly women with hypertension, the most vulnerable population group to health, could have an important influence on exercise intervention studies. We tried to determine whether an exercise pre- 

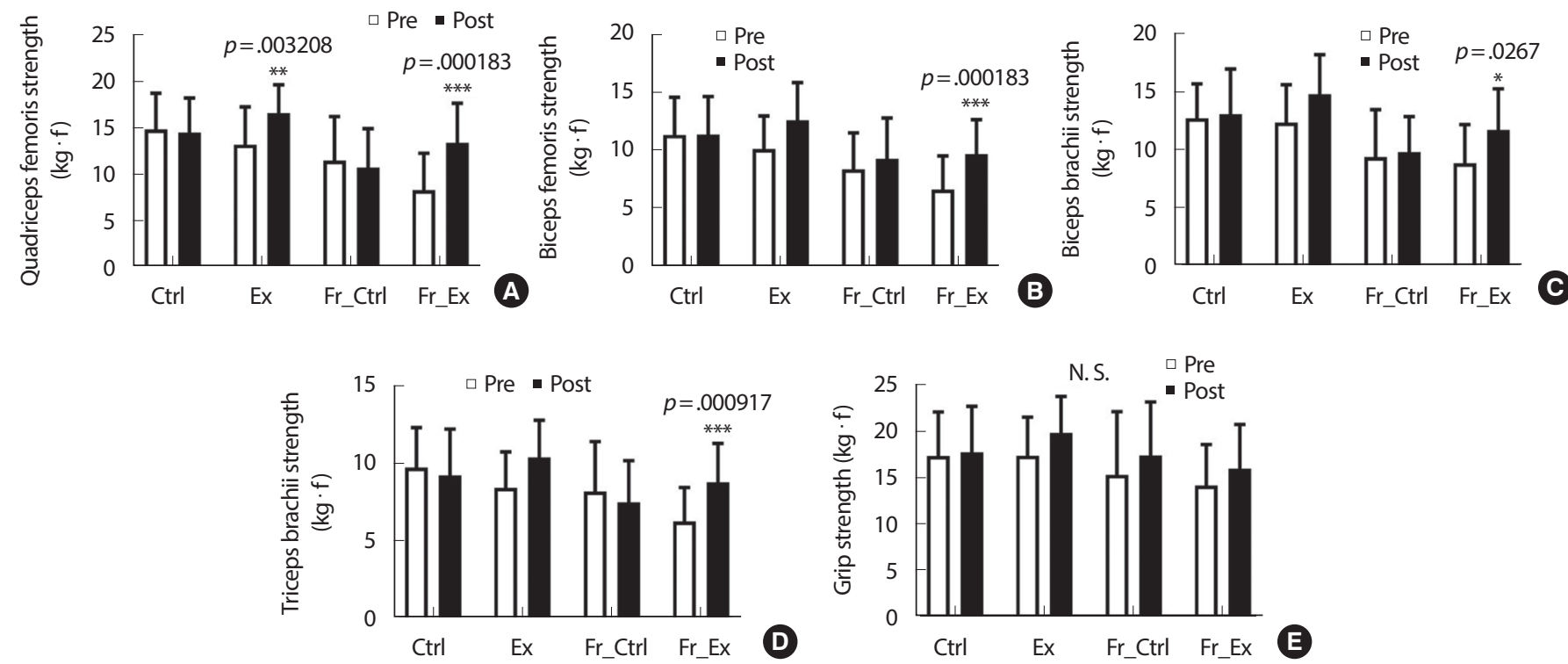

Fig. 4. Muscular strength of pre- and post-exercise intervention in hypertensive female elderly. Data are presented mean \pm SD. N. S. $=$ no significant difference. ${ }^{*} p<.05,{ }^{* *} p<.01$ and ${ }^{* * *} p<.001$, pre vs. post.

scription or exercise program should be designed after additionally classifying elderly women with hypertension by frailty level through the results of this study.

Body composition, blood pressure, fasting blood glucose level (include $\mathrm{HbA}_{\mathrm{Ic}}$ ) and blood lipid profile in this study did not change even though exercise intervention was performed in elderly women with hypertension. The main reason these results appeared may be that the obesity was not set as a criterion for classifying the subjects in each group, and also all subjects had no history of diabetes mellitus and dyslipidemia. Therefore, factors other than BP in elderly women with hypertension did not show significant improvement by exercise, but could be interpreted as maintaining normal ranges.

Since the exercise-induced energy expenditure and food intake were not measured, there is no data can clearly determine why body weight or body composition did not change. There is no data that can confirm the cause of this results because exercise-induced energy expenditure and food intake was not measured. However, it can be difficult to induce weight loss, reduce blood pressure, reduce fasting blood glucose levels and blood lipid profiles because diet restrictions are not applied. These interpretations and judgments are based on research results that show that exercise does not have the effect of pure weight loss without dietary restrictions $[13,14]$. In other words, it means that exercising with dietary restrictions can help to lose weight. This is supported by the result that significantly weight loss was induced by dietary restriction during 8-week exercise intervention in obese women [14].
Although our study did not significantly decrease or improvement in BP, fasting blood glucose level and blood lipid profile, these results do not mean that body composition and various obesity related factors cannot be improved in elderly women with hypertension. Several studies have confirmed that exercise can decrease BP [15-17], fasting blood glucose level [18] and improve blood lipid profile [19]. Also, apart from this, exercise itself is an important factor that can improve the quality of life of the elderly [20]. However, the exercise program in this study did not induce improvement of several factors. What can be clearly stated based on our research data is that there is no interaction between exercise and frailty levels on BP and fasting blood glucose levels.

We have identified interesting findings (Fig. 3A and B). In a study of non-hypertensive elderly people over 75 years, the RGT record improved within a short period compared to 30-s CST, and the improvement was also greater [21]. However, in our study results, it was confirmed that the 30-s CST record increased significantly after exercise intervention compared to before exercise intervention regardless of the level of frailty (Ex, $p<.01 ;$ Fr_Ex, $p<.0001)$. These results is interesting because RGT and 30-s CST are important tools for frailty diagnosis. RGT is a tool to analyze agility and dynamic balance, and 30-s CST is a tool to indicate lower body strength and muscular endurance. Therefore, it is thought that in elderly women with hypertension, the lower body muscle strength and muscular endurance relatively early improve after exercise intervention than agility and dynamic balance improve. We estimated several reasons for the improvement of RGT and 30-s CST and different results 

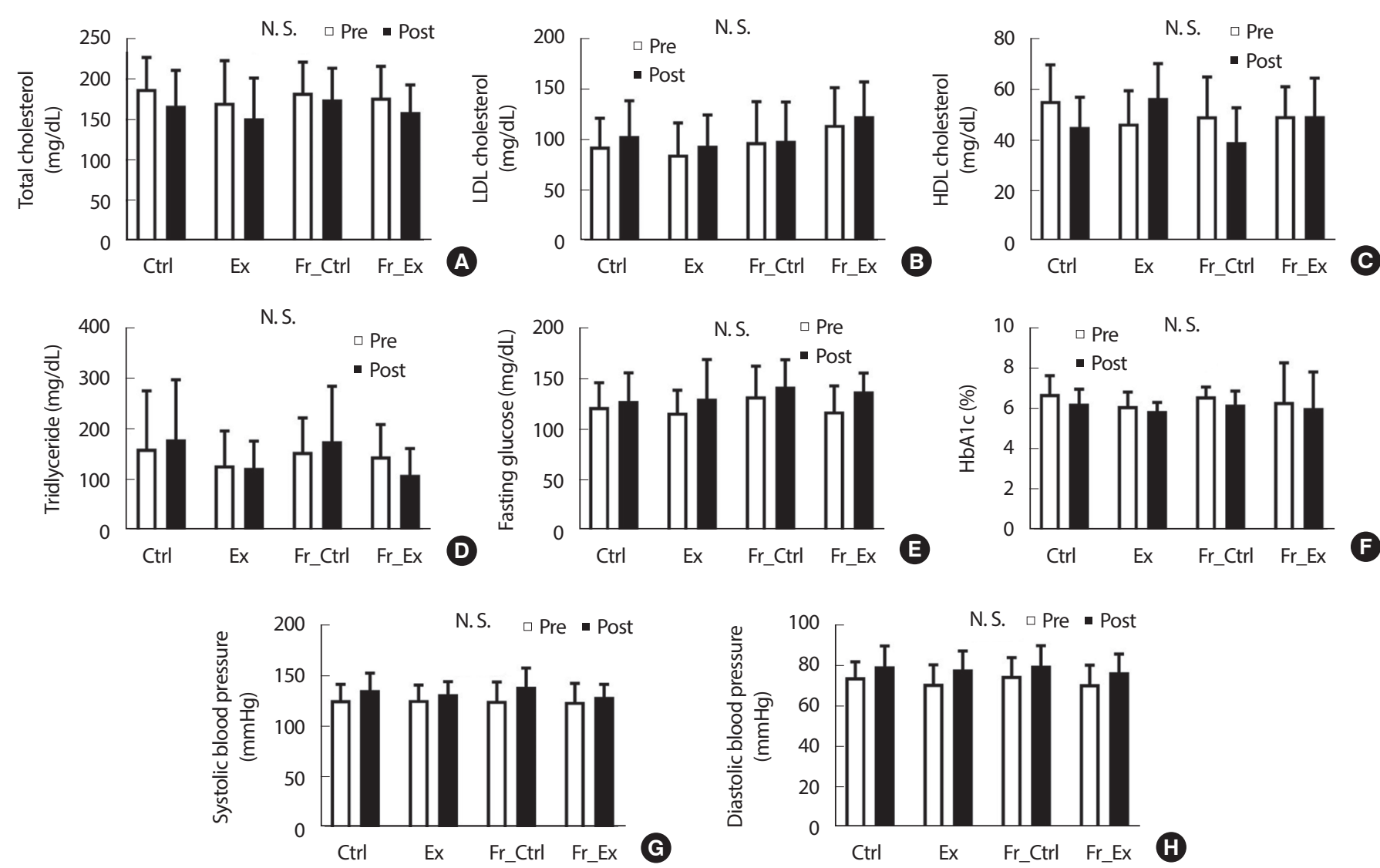

Fig. 5. Blood pressure, fasting blood glucose level and blood lipid profile of pre- and post-exercise intervention in hypertensive female elderly. Data are presented mean \pm SD. N. S. = no significant difference. $L D L=$ low-density lipoprotein. $H D L=$ high-density lipoprotein.

in the elderly women with hypertension in this study, unlike previous study. We pondered why the improvement of RGT and 30-s CST appeared in this study differently from previous study. In the case of hypertensive female elderly, it is difficult to improve agility and dynamic balance through exercise compared to non-hypertensive elderly. The reason for this is considered to be that the level of agility and dynamic balance, which are skill-related physical fitness, is very closely related to aging in the elderly women with hypertension compared to the normal elderly. These speculations can be more clearly demonstrated only by comparing them with the subjects of this study in elderly women without hypertension. However, we analyzed the correlation between RGT (Fig. 6A), 30-s CST (Fig. 6B) and two-minute walk test (Fig. 6C) records of this study and age as a second best practice. In the statistical analysis results, it was judged that the higher the correlation with age, the lower the level of physical fitness components related to each physical fitness test. As a results, it was confirmed that the correlation between RGT $(p<.0001)$ records and age was much greater than that of the 30-s CST $(p<.05)$, the arm curl repetitions $(p<.05)$ and the two-minute walk test $(p<.05)$ (Fig. 6). Therefore, it was judged that the agility and dynamic balance of the subjects of this study were very low. Also, since the correlation with age is very high, it was judged as a physical fitness components that is difficult to improve independently of age. From the results of the chair stretch test and back scratch test, flexibility also seems to be a difficult factor to improve through exercise for elderly women with hypertension, such as agility and dynamic balance (Fig. 3C and F).

In elderly women with hypertension, quadriceps femoris strength improved after exercise intervention regardless of the level of frailty (Fig. 4A). The muscle strength of quadriceps femoris increased significantly after exercise intervention regardless of the level of frailty, but the difference between pre- and post-intervention was statistically clear in Fr_Ex ( $p=.000183)$ compared Ex ( $p=.003208)$. The muscle strength of biceps femoris, biceps brachii and triceps brachii was significantly improved after exercise intervention in only Fr_Ex compared to before exercise intervention (Fig. 3B, C, and D). These results show that the effect of exercise for elderly women with hypertension has different effects on muscle strength depending on the level of frailty. In particular, the statistical 

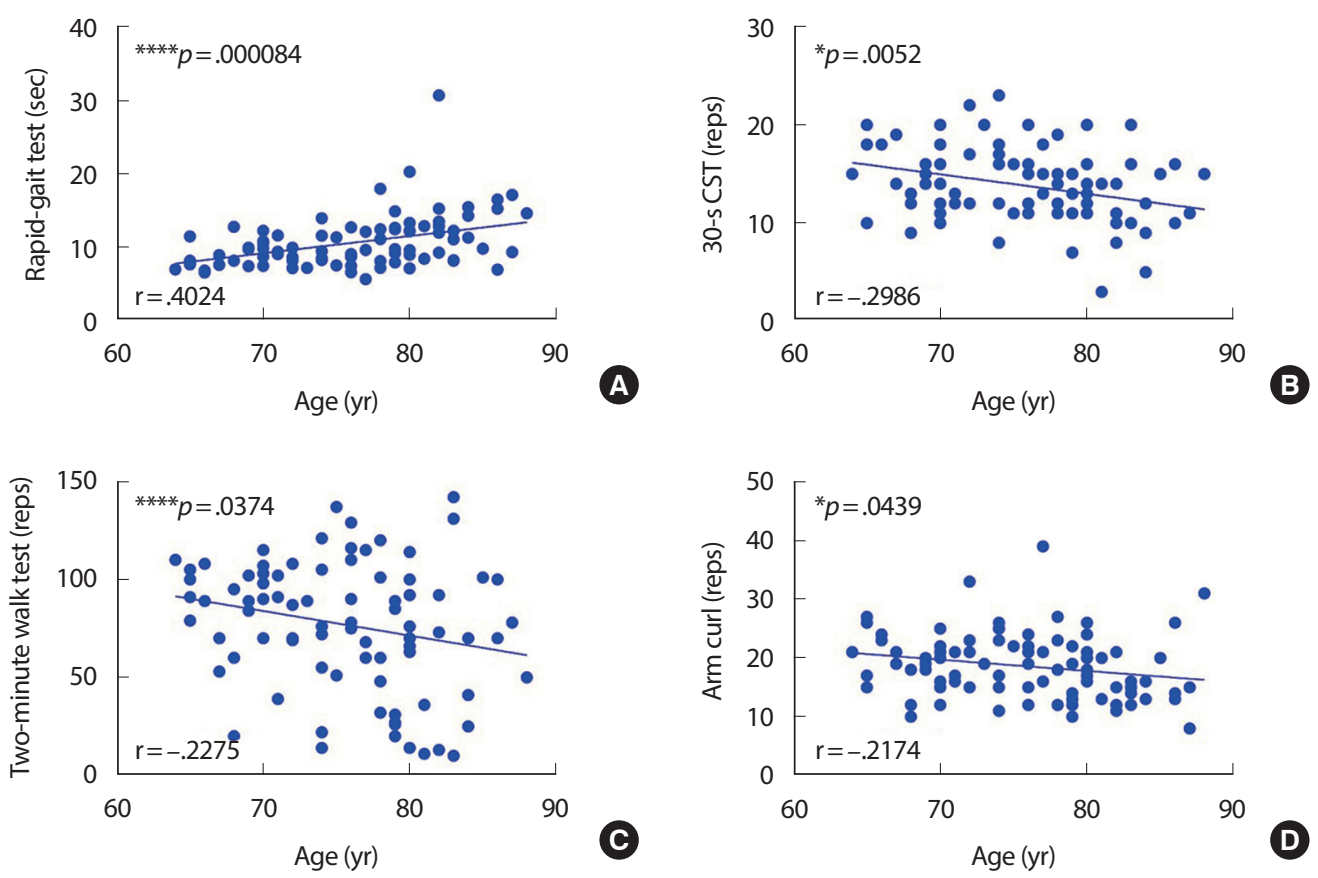

Fig. 6. Correlation between age and our frailty test record and some senior fitness test record. ${ }^{*} p<.05$ and ${ }^{* * * *} p<.0001$, age $\times$ variable. $r=$ Pearson's correlation coefficient.

difference more clearly in posterior muscles than anterior muscles (biceps brachii vs. triceps brachii in Fr_Ex, $p<.05$ vs. $p<.001$; quadriceps femoris, Ex vs. Fr_Ex, $p<.01$ vs. $p<.001$; quadriceps femoris in Fr_Ex, $p<.0001)$. Taken together, it is possible to speculate that among the elderly women with hypertension, the frailty subjects have a lot of weakened lower- and upper-limb muscle, and especially the posterior muscles. In any case, the classification of the level of frailty in hypertensive elderly women causes a significant difference in the results of exercise intervention.

\section{CONCLUSION}

The additional classification according to the level of frailty in hypertensive female elderly can have a significant effect on the degree of muscle strength improvement by exercise intervention. In addition, this study newly discovered that the improvement of 30-s CST record in the hypertensive elderly women appeared faster than the improvement of the RGT record independently of the level of frailty.

\section{CONFLICT OF INTEREST}

The authors declare that they do not have conflict of interest.

\section{AUTHOR CONTRIBUTION}

Conceptualization: J Yoo, JS Kim; Data curation: K Baek, MC Lee, T Jeon; Formal analysis: K Baek, MC Lee, T Jeon; Funding acquisition: J Yoo, JS Park; Methodology: T Jeon, HY Moon; Project administration: T Jeon, JS Kim; Visualization: K Baek, MC Lee; Writing-original draft: K Baek, MC Lee, JS Kim; Writing-review \& editing: K Baek, J Yoo, JS Park, HY Moon, JS Kim.

\section{ORCID}

Kyung-Wan Baek https://orcid.org/0000-0002-8445-3773

Min Chul Lee https://orcid.org/0000-0003-4440-7462

Tae-Bong Jeon https://orcid.org/0000-0001-5902-2579

Jun-Il Yoo https://orcid.org/0000-0002-3575-4123

Jin Sung Park https://orcid.org/0000-0002-6284-9566

Hyo Youl Moon https:/orcid.org/0000-0001-8207-1301

Ji-Seok Kim https://orcid.org/0000-0002-3023-1999

\section{REFERENCES}

1. Benetos A, Petrovic M, Strandberg T. Hypertension management in older and frail older patients. Circ Res. 2019;124(7):1045-60. 
2. Vasan RS, Beiser A, Seshadri S, Larson MG, Kannel WB, et al. Residual lifetime risk for developing hypertension in middle-aged women and men: The Framingham Heart Study. JAMA. 2002;287(8):1003-10.

3. Choi HM, Kim HC, Kang DR. Sex differences in hypertension prevalence and control: analysis of the 2010-2014 korea national health and nutrition examination survey. PLoS One. 2017;12(5):e0178334.

4. Barili A, Corralo VDS, Cardoso AM, Manica A, Bonadiman B, et al. Acute responses of hemodynamic and oxidative stress parameters to aerobic exercise with blood flow restriction in hypertensive elderly women. Mol Biol Rep. 2018;45(5):1099-109.

5. Cunha RM, Vilaca-Alves J, Noleto MV, Silva JS, Costa AM, et al. Acute blood pressure response in hypertensive elderly women immediately after water aerobics exercise: a crossover study. Clin Exp Hypertens. 2017;39(1):17-22.

6. Dantas FF, Brasileiro-Santos Mdo S, Batista RM, do Nascimento LS, Castellano LR, et al. Effect of strength training on oxidative stress and the correlation of the same with forearm vasodilatation and blood pressure of hypertensive elderly women: a randomized clinical trial. PLoS One. 2016;11(8):e0161178.

7. Gill TM, Baker DI, Gottschalk M, Peduzzi PN, Allore H, et al. A program to prevent functional decline in physically frail, elderly persons who live at home. N Engl J Med. 2002;347(14):1068-74.

8. Whitworth JA, World Health Organization ISoHWG. 2003 World Health Organization (WHO)/International Society of Hypertension (ISH) statement on management of hypertension. J Hypertens. 2003; 21(11):1983-92.

9. Langhammer B, Stanghelle JK. The senior fitness test. J Physiother. 2015;61(3):163.

10. Buckinx F, Croisier JL, Reginster JY, Dardenne N, Beaudart C, et al. Reliability of muscle strength measures obtained with a hand-held dynamometer in an elderly population. Clin Physiol Funct Imaging. 2017;37(3):332-40

11. Mentiplay BF, Perraton LG, Bower KJ, Adair B, Pua YH, et al. Assessment of lower limb muscle strength and power using hand-held and fixed dynamometry: a reliability and validity study. PLoS One. 2015; 10(10):e0140822.
12. Kim CR, Jeon YJ, Kim MC, Jeong T, Koo WR. Reference values for hand grip strength in the south korean population. PLoS One. 2018;13 (4):e0195485.

13. Racette SB, Schoeller DA, Kushner RF, Neil KM, Herling-Iaffaldano K. Effects of aerobic exercise and dietary carbohydrate on energy expenditure and body composition during weight reduction in obese women. Am J Clin Nutr. 1995;61(3):486-94.

14. Kempen KP, Saris WH, Westerterp KR. Energy balance during an 8-wk energy-restricted diet with and without exercise in obese women. Am J Clin Nutr. 1995;62(4):722-9.

15. Mota MR, de Oliveira RJ, Dutra MT, Pardono E, Terra DF, et al. Acute and chronic effects of resistive exercise on blood pressure in hypertensive elderly women. J Strength Cond Res. 2013;27(12):3475-80.

16. de Sousa EC, Abrahin O, Ferreira ALL, Rodrigues RP, Alves EAC, et al. Resistance training alone reduces systolic and diastolic blood pressure in prehypertensive and hypertensive individuals: meta-analysis. Hypertens Res. 2017;40(11):927-31.

17. Ruangthai R, Phoemsapthawee J. Combined exercise training improves blood pressure and antioxidant capacity in elderly individuals with hypertension. J Exerc Sci Fit. 2019;17(2):67-76.

18. Blumenthal JA, Sherwood A, Gullette EC, Babyak M, Waugh R, et al. Exercise and weight loss reduce blood pressure in men and women with mild hypertension: effects on cardiovascular, metabolic, and hemodynamic functioning. Arch Intern Med. 2000;160(13):1947-58.

19. Ammar T. Effects of aerobic exercise on blood pressure and lipids in overweight hypertensive postmenopausal women. J Exerc Rehabil. 2015;11(3):145-50.

20. Dechamps A, Diolez P, Thiaudiere E, Tulon A, Onifade C, et al. Effects of exercise programs to prevent decline in health-related quality of life in highly deconditioned institutionalized elderly persons: a randomized controlled trial. Arch Intern Med. 2010;170(2):162-9.

21. Gill TM, Baker DI, Gottschalk M, Peduzzi PN, Allore H, et al. A prehabilitation program for the prevention of functional decline: effect on higher-level physical function. Arch Phys Med Rehabil. 2004;85(7): 1043-49. 